\title{
Homochiral Skyrmionic Bubbles in Exfoliated 2D Van Der Waals Cr2Ge2Te6
}

Myung-Geun Han ${ }^{1}$, Joseph Garlow ${ }^{1}$, Yimei Zhu ${ }^{1}$, Huiqin Zhang ${ }^{2}$, Yu Liu ${ }^{1}$, Donald DiMarzio ${ }^{3}$, Cedomir Petrovic $^{1}$ and Deep Jariwala ${ }^{2}$

${ }^{1}$ Brookhaven National Laboratory, Upton, New York, United States, ${ }^{2}$ University of Pennsylvania, Philadelphia, Pennsylvania, United States, ${ }^{3}$ Northrop Grumman Corporation, Redondo Beach, California, United States

The recent discovery on long-range ferromagnetic order down to atomic layers provides an important degree of freedom in engineering two-dimensional (2D) materials and their heterostructure devices for spintronics, valleytronics and magnetic tunnel junction switches [1-3]. In addition to simple 2D ferromagnetism, topological spin textures, such as vortices, skyrmions, and monopoles, are an important ingredient for designing and realizing novel topologically driven quantum states in all 2D heterostructures. Using direct imaging by cryo-Lorentz transmission electron microscopy we report that topologically nontrivial magnetic-spin states, skyrmionic bubbles, can be realized in exfoliated insulating 2D van der Waals (vdW) $\mathrm{Cr}_{2} \mathrm{Ge}_{2} \mathrm{Te}_{6}(\mathrm{CGT})$.

$2 \mathrm{D} v d W$ CGT is a ferromagnetic insulator with an indirect band gap of $\sim 0.74 \mathrm{eV}$. Ferromagnetism arises from the super exchange interaction between nearest-neighbor $\mathrm{Cr}^{3+}$ ions linked by Te ligands through nearly 90o angles (Figure 1), which shows a monoaxial anisotropy with the easy axis along the c-axis. The ferromagnetically ordered $\mathrm{Cr}$ spins aligned along the c-axis are found to be stable down to double atomic-layers, as recently demonstrated [3]. Similar to bubble domains in thin films with an anisotropy ratio $\mathrm{Q}>>1$ and an easy axis perpendicular to the film plane, stripe domains separated by Bloch walls spontaneously develop without external magnetic field (Fig. 2a). Cylindrical-shape bubbles emerge before the field-induced saturation as a metastable state (Fig. 2b), similar to emergence of skyrmions in the vicinity of ferromagnetically poled states in chiral magnets. The lowest energy state of a bubble is characterized by a cylindrical Bloch wall with a single rotation sense. However, due to the lack of symmetry breaking DMI, internal spin structures, such as Bloch lines and points, can occur within the Bloch walls of stripe domains and bubbles, leading to a variety of topological configurations that are classified with their distinctive topological charges [4-5]; type-I without Bloch lines or points, and typeII with Bloch lines or points [5].

Similar to magnetic bubble materials, hexagonally packed nanoscale bubble lattices emerge in the ab plane by field (50 mT) cooling (Fig. 2d-e) [6]. Despite a range of topological spin textures arising due to pair formation and annihilation of Bloch lines, bubble lattices with single chirality are prevalently observed in our study (Fig. 2f). Our observation of topologically nontrivial homochiral skyrmionic bubbles in exfoliated vdW materials provides a new avenue for novel quantum states in atomically-thin insulators for magneto-electronic and quantum devices. 

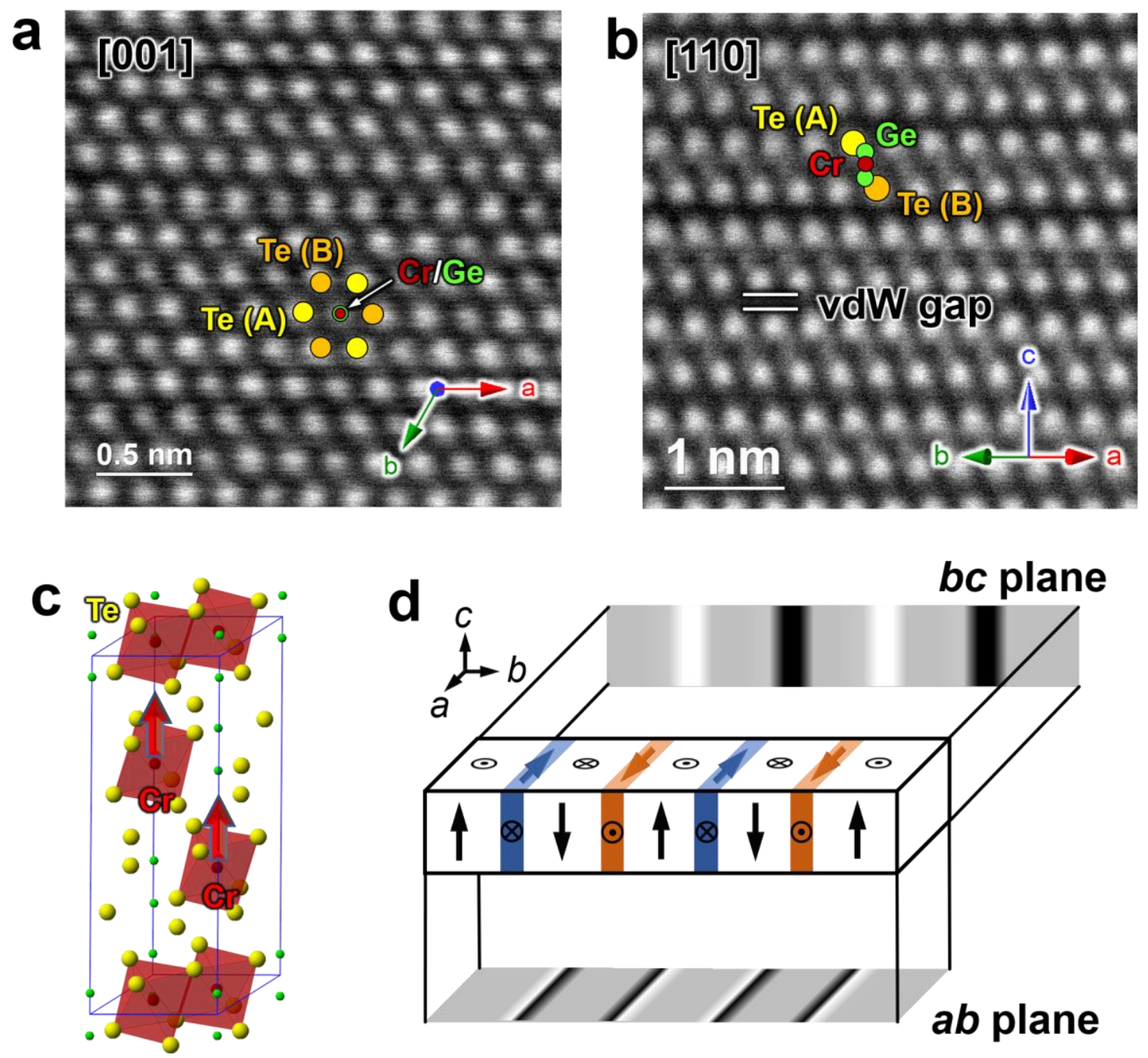

Figure 1. Atomic structure of $2 \mathrm{D}$ vdW $\mathrm{Cr} 2 \mathrm{Ge} 2 \mathrm{Te} 6$ and stripe domains due to uniaxial anisotropy. $a-b$ HAADF STEM images along the [001] and [110] directions, respectively. c Cr2Ge2Te6 unit cell showing CrTe6 octahedra and Ge pairs. Red arrows depict the spin moments for $\mathrm{Cr}$ atoms along the c-axis. $\mathrm{d}$ Schematic of stripe domains due to uniaxial anisotropy and their LTEM contrasts projected on bc and ab planes. Stripe domains with antiparallel spins are separated by Bloch walls. 


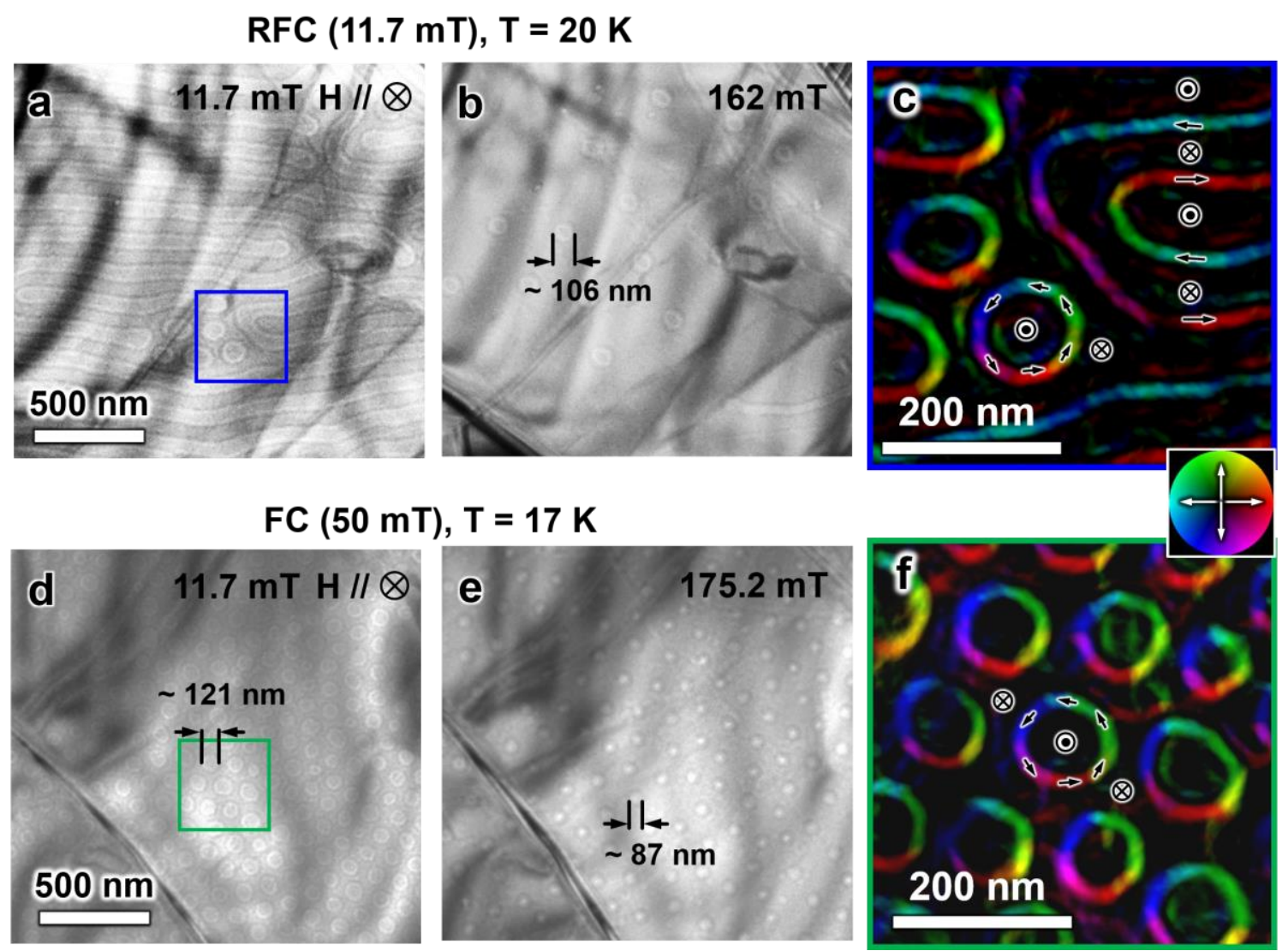

Figure 2. a-b, LTEM images showing magnetic-field-driven transitions from stripes (a) to skyrmionic bubbles (b) by external magnetic field in residual-field $(11.7 \mathrm{mT})$ cooled sample. External magnetic field (H) was applied along the imaging direction (i.e., along the c-axis, pointing into the ab plane of exfoliated flakes). c Magnetization map obtained by the transport-of-intensity equation (TIE) analysis of focal series of LTEM data from the area indicated with blue box in a. d-e, LTEM images showing hexagonally packed skyrmionic bubbles in field-cooled $(50 \mathrm{mT})$ sample. When external magnetic field is increased to 175.2 $\mathrm{mT}$, the skyrmionic bubble size decreases. f Magentization map showing single-chiral skyrmionic bubbles.

This work was supported by the US Department of Energy, Basic Energy Sciences, Materials Sciences and Engineering Division (Contract No. DE-SC0012704). D.J. and H.Z. acknowledge support from Northrop Grumman. Research carried out, in part, at the Center for Functional Nanomaterials, Brookhaven National Laboratory.

References

[1] K. S. Burch, D. Mandrus, and J. Park, Nature 563, 4 (2018).

[2] B. Huang, et al., Nature 546, 270 (2017).

[3] C. Gong, et al., Nature 546, 265 (2017).

[4] R. Schäfer and A. Hubert, Magn. Domains 40 (159), 108-157 (2000).

[5] J. C. Loudon, et al., Adv. Mater. 31, 16 (2019).

[6] M.-G. Han, et al., Nano Lett. 19, 7859-7865 (2019). 